\title{
Investigation of Magnetic Properties of Magnetic Poly (glycidyl methacrylate) Microspheres: Experimental and Theoretical
}

\author{
Hoang Thanh Nguyen $\mathbb{D}^{1,2}$ and Tuan Manh Nguyen $^{3}$ \\ ${ }^{1}$ Ho Chi Minh City Institute of Physics, Vietnam Academy of Science and Technology, Ho Chi Minh 70000, Vietnam \\ ${ }^{2}$ Graduate University of Science and Technology, Vietnam Academy of Science and Technology, Ha Noi 100000, Vietnam \\ ${ }^{3}$ Institute of Applied Materials Science, Vietnam Academy of Science and Technology, Ho Chi Minh 70000, Vietnam
}

Correspondence should be addressed to Hoang Thanh Nguyen; nthoang@hcmip.vast.vn

Received 9 November 2020; Revised 2 June 2021; Accepted 14 June 2021; Published 25 June 2021

Academic Editor: José António Fonseca de Oliveira Correia

Copyright (C) 2021 Hoang Thanh Nguyen and Tuan Manh Nguyen. This is an open access article distributed under the Creative Commons Attribution License, which permits unrestricted use, distribution, and reproduction in any medium, provided the original work is properly cited.

\begin{abstract}
Biocompatible magnetic poly (glycidyl methacrylate) microsphere is a novel nanocomposite with a myriad of promising bioapplications. Investigation of their characteristics by experimental analysis methods has also been carried out in the past. However, a survey of the magnetic anisotropy constant has not been mentioned and the influence of the poly (glycidyl methacrylate) polymer matrix on the $\mathrm{Fe}_{3} \mathrm{O}_{4}$ magnetite nanoparticles embedded inside has also not been discussed. Moreover, the accurate characterization of the magnetite nanoparticle size distribution remains challenging. In this paper, we present an effective approach was used to solve these problems. First of all, we combine both experiment and theory to estimate the effective magnetic anisotropy constant. Besides that, we implement an accurate method to determine magnetite nanoparticle size distribution in the magnetic poly (glycidyl methacrylate) microspheres composite nanomaterial.
\end{abstract}

\section{Introduction}

During the last decade, $\mathrm{Fe}_{3} \mathrm{O}_{4}$ magnetite nanoparticles (MNPs) have been used in large number of fields, such as water treatment $[1,2]$, image diagnosis (magnetic resonance imaging (MRI) contrast agent) [3], cancer therapy (magnetic hyperthermia mediator, targeted drug, or gene delivery vehicle) $[4,5]$, separating cells, and capturing proteins/ biomolecules [6]. In these various biomedical applications, the essential issues must mention that the MNPs will be accumulated to large cluster under the different agents (strong magnetic dipole-dipole interaction, London-van der Waals attraction) [7]. In order to improve bioavailability and minimize adverse effects, reduce the interparticle interaction, and control the size of the MNPs, the biocompatible polymers with their flexible functionalization are widely used as a matrix to embed the MNPs inside. Numerous polymer materials, both natural and synthetic, including chitosan [8], carboxymethyl cellulose [1], and dextran and polymethyl methacrylate (PMMA) $[9,10]$, have been investigated. Besides that, poly (glycidyl methacrylate) (PGMA) is also well known as a self-assembly, biodegradability, and $\mathrm{pH}$ sensitive polymer. The morphologies of PGMA derivatives are very unique, which is possible to create complex structures by way of self-assembly, such as micelle (spherical, cylindrical, etc.), reversal micelles (RM), capsule, and nanoparticle [11]. In addition, the different triggers of internal and external factors, such as length and ratio of the hydrophobic fraction, $\mathrm{pH}$, solvent composition, and temperature, can change their morphology [12]. In addition, the advantage of the poly (glycidyl methacrylate) (PGMA) is being hydrophilic, as epoxy groups can be easily modified to carry a variety of reactive functional groups like $\mathrm{NH}_{2}, \mathrm{COOH}$, and $\mathrm{SH}$, which can be conjugated with biomolecules (antibodies, peptides, and drugs) [13], and stability (no aggregation) in various biological media. Therefore, the PGMA polymer microsphere embeds the MNPs showing great potential in nanomedicine, biotechnology, and molecular biology [14]. However, this biocompatible polymer may interact with the surface atoms of 
the MNPs embedded inside and form a magnetically disordered layer or nonmagnetic layer [15]. As a result, it contributes to the influence on the particles size distribution and the magnetic anisotropy, which are the crucial parameters in numerous applications [16].

The most successful methods at obtaining well-dispersed MNPs with narrow size distribution and uniform shape are microemulsion, polyol methods, and homogeneous precipitation or coprecipitation technique. The latter is the most common, easiest, versatile, convenient, and most efficient synthesis method used to obtain the MNPs, not only for laboratory purposes but also for industrial scale. The advantages of this method are that the reaction occurs at low temperatures and the obtained MNPs have fewer impurities compared to the microemulsion/solvothermal method [17]. However, the downside is that the particle size distribution cannot be controlled [18, 19]. Anushree et al. used the coprecipitation method to synthesize the MNPs; at the same time the oxidation-precipitation method was also used to prepare carboxymethyl cellulose capped MNPs for various applications. These positive results were reported at $[1,20]$. To fabricate the magnetic polymer microsphere materials, various formulas can be used. First of all, the most simple method that must be mentioned is that the magnetic particles and polymer materials are synthesized separately and then mixed together; thence the magnetic nanoparticles are formed inside the polymer matrix. Moreover, there are other common methods to obtain magnetic polymer beads, such as suspension polymerization, microemulsion polymerization, and free-emulsion polymerization in the presence of magnetic nanoparticles $[11,21,22]$. A disadvantage of these methods is that the magnetic microspheres obtained do not have a uniform size and shape. However, the method used in this work is simple, repeatable, and suitable for the preparation of monodisperse multifunctional microspheres with desired compact size.

As we have known, the magnetic properties of the MNPs are strongly affected by intrinsic parameters such as lattice constant, surface energy, and magnetic anisotropy, which depends on particle size and shape [23]. Additionally, for the bioapplications like magnetic hyperthermia (MHT) applications, the magnetic anisotropy constant plays a key role [24-28]. However, the particle size determination methods of the MNPs embedded inside the magnetic PGMA microsphere are, for example, observing with transmission electron microscopy (TEM) or using Scherrer equation from X-ray diffraction (XRD) results, which have not been highly reliable and fully reflect essential information [29]. Specifically, TEM images cannot observe fully a large amount of the MNPs embedded inside the PGMA microspheres. Therefore the particle size distribution is only local, which cannot be representative of the whole. Besides, the results obtained from the Scherrer equation are only average crystallite sizes, which can be considered as particle sizes when all particles are single crystal. Not only that, but also size determination from powder XRD, with the presence of the polymer matrix, rises the noise of the patterns, which has led to an increase in the false results. To the best knowledge of the authors, the magnetic anisotropy constant value, influence of PGMA polymer matrix to the MNPs, and the particle size distribution function determination of the MNPs inside the PGMA microspheres nanocomposite material are not studied so far. The aim of this study is to fabricate the magnetic poly (glycidyl methacrylate) (M-PGMA) microspheres for biomedical applications, which are prepared by in situ precipitation of the MNPs inside the PGMA polymer microspheres. On the one hand, experimental analysis methods like XRD, scanning electron microscopy (SEM), Fourier-transform infrared spectroscopy (FTIR), and vibrating sample magnetometer (VSM) were used to investigate the characteristics of the M-PGMA microspheres. On the other hand, the theoretical approach, law of approach to saturation magnetization (LAS) principle, was also used to estimate the effective magnetic anisotropy constant $\left(K_{\text {eff }}\right)$ of the synthesized material at room temperature $(300 \mathrm{~K})$ and compared it to the obtained result from the relation of $K_{\text {eff }}$ with blocking temperature $\left(T_{\mathrm{B}}\right)$ in zero-field-cooled/field-cooled (ZFC/FC) magnetization curves measurement. In addition, using the fitting of experimental magnetization curve data to the Langevin function enables determination of the median particle size, the corresponding standard deviation, and the particle size distribution function of the MNPs embedded inside the M-PGMA microspheres.

\section{Materials and Methods}

2.1. Materials. The materials used are monomer glycidyl methacrylate (GMA) (>97\%) from Merck, 2,2'-azobis-isobutyronitrile (AIBN) from Sigma-Aldrich, poly (vinyl pyrrolidone) (PVP), ferric chloride hexahydrate $\left(\mathrm{FeCl}_{3} \cdot 6 \mathrm{H}_{2} \mathrm{O}\right)$, ferrous chloride tetrahydrate $\left(\mathrm{FeCl}_{2} \cdot 4 \mathrm{H}_{2} \mathrm{O}\right)$, ammonium hydroxide $(25 \%)$, ethylene diamine $\left(\mathrm{C}_{2} \mathrm{H}_{4}\left(\mathrm{NH}_{2}\right)_{2}\right.$, sodium hydroxide $(\mathrm{NaOH})$, pure nitrogen gas $\left(\mathrm{N}_{2}\right)$, and deionized (DI) water.

\subsection{Fabricating the M-PGMA Microspheres}

2.2.1. Polymerization of Monodisperse PGMA Microspheres. $3 \mathrm{~g}$ PVP, $152.7 \mathrm{ml}$ ethanol, and $13.5 \mathrm{ml}$ DI water were put into the reaction flask and stirred, and $11.2 \mathrm{ml}$ monomer glycidyl methacrylate (GMA) and $0.24 \mathrm{~g}$ AIBN were added to the flask. Nitrogen gas $\left(\mathrm{N}_{2}\right)$ was pumped into the solution to remove the air from the system for 20 hours at $80^{\circ} \mathrm{C}$ and then it was refrigerated to $15^{\circ} \mathrm{C}$. Then, we washed the sample several times with ethanol and DI water. Finally, the sample was dried by lyophilization.

Preparation of PGMA microspheres with amino groups (amino-PGMA): ethylene diamine was used to modify the chemical bond of PGMA microspheres, reacting with epoxy groups to form functional amino groups. Dried PGMA sample $(7 \mathrm{~g})$ was mixed with DI water $(75 \mathrm{ml})$ and ethylenediamine $(75 \mathrm{ml})$ at $80^{\circ} \mathrm{C}$ for 10 hours. After the reaction, the particles were separated and washed several times with DI water and then dried by lyophilization. 
2.2.2. Preparation of M-PGMA Microspheres. The aminoPGMA dried sample $(8 \mathrm{~g})$ was dispersed into DI water $(75 \mathrm{ml})$ and cooled down to $15^{\circ} \mathrm{C} \cdot 0.2 \mathrm{M} \mathrm{FeCl} \cdot 6 \mathrm{H}_{2} \mathrm{O}$ and $0.1 \mathrm{M} \mathrm{FeCl}_{2} \cdot 4 \mathrm{H}_{2} \mathrm{O}$ in ratio $1: 1$ were dissolved in DI water $(80 \mathrm{ml})$ and cooled down to $15^{\circ} \mathrm{C}$ and then mixed and stirred with the solution containing the amino-PGMA. Stirring was done at $800 \mathrm{rpm}$ for about 30 minutes, and then the ammonia solution (25\%) was added by pipette. The temperature is maintained at $80^{\circ} \mathrm{C}$ for 60 minutes for the continuation of the reaction. The particles were washed several times with DI water to remove excessive ammonia. Finally, the sample was dried by lyophilization.

Fabricating bare $\mathrm{Fe}_{3} \mathrm{O}_{4}$ MNPs under the same experimental conditions for comparison: $\mathrm{Fe}_{3} \mathrm{O}_{4}$ nanoparticles were synthesized using the coprecipitation method. $0.2 \mathrm{M}$ $\mathrm{FeCl}_{3} \cdot 6 \mathrm{H}_{2} \mathrm{O}$ and $0.1 \mathrm{M} \mathrm{FeCl}_{2} \cdot 4 \mathrm{H}_{2} \mathrm{O}$ were dissolved in $80 \mathrm{ml}$ of DI water, followed then by stirring for 20 minutes, dripping the ammonia solution $(25 \%)$ by pipette, and continuously stirring for 60 minutes at $80^{\circ} \mathrm{C}$ for a complete occurrence of the reaction. The particles were washed several times with DI water and then dried by vacuum oven at $80^{\circ} \mathrm{C}$ in 8 hours.

Overview diagram of the experiment is shown in Figure 1.

2.3. The Law of Approach to Saturation (LAS) Principle. The law of approach to saturation (LAS) is a well-known mathematical model for describing the behavior of ferromagnets at strong magnetic fields $\left(H \gg H_{c}\right)$, which has the additional advantage that it is connected with the anisotropy constant of the material through one of the terms in the mathematical expression. The LAS describes the dependence of the magnetization $(M)$ on the applied magnetic field $(H)$ at a certain field, which is near the saturation magnetization $M_{\mathrm{s}}$ $\left(H \gg H_{c}\right)$. According to the LAS model, the magnetization near the saturation $\left(M_{\mathrm{s}}\right)$ can be expressed as follows [30]:

$$
M=\mathrm{M}_{\mathrm{s}}\left(1-\frac{a}{H}-\frac{b}{H^{2}}-\cdots\right),
$$

where $M_{\mathrm{s}}$ is the saturation magnetization, $H$ is the applied magnetic field, and $b$ is the parameter containing the magnetic anisotropy constant. The term a/ $H$ was interpreted as the result of the stress field and nonmagnetic inclusions, which was explained by Brown and Neel [31]; therefore, we can neglect the a/H term and the $H^{n}$ terms with $n>2$ in equation (1) [32]. Equation (1) can be rewritten as

$$
M=M_{s}\left(1-\frac{b}{H^{2}}\right) \text {. }
$$

According to [33], the effective anisotropy constant of material can be estimated based on a relationship between parameter $b$ and the $K_{\text {eff }}$ as follows:

$$
K_{\text {eff }}=\mu_{0} M_{s} \sqrt{\frac{15 b}{4}}
$$

where $K_{\text {eff }}$ is the effective anisotropy constant of material and $\mu_{0}$ is the magnetic permeability.
2.4. Particle Size Determination from Magnetization Curve. As we have known, the magnetization curves of superparamagnetic nanoparticles (assuming that they are all of the same size) at room temperature $(300 \mathrm{~K})$ can be expressed by Langevin function [34]:

$$
L=\operatorname{costh}(b)-\frac{1}{\mathrm{~b}}
$$

where $b=\mu H / k_{\mathrm{B}} T, \mu=M_{\mathrm{sb}} V$ is the particle magnetic moment, $V$ is the particle volume, and $M_{\mathrm{sb}}$ is the saturation magnetization of the bulk material. The saturation magnetization of superparamagnetic nanoparticles is $M_{\mathrm{s}}=\varepsilon M_{\mathrm{sb}}$ with $\varepsilon$ being the volumetric particle packing fraction.

In practice, a magnetic nanoparticle system never has exactly the same size, which often follows a distribution function of particle sizes. The log-normal distribution is one of the most common distribution functions used to investigate the distribution of particle size [35]. Many works have demonstrated that the log-normal size distribution is known to be the natural distribution of nanoparticle sizes $[30,36]$, which is written as follows:

$$
f(D)=\frac{1}{\sqrt{2 \pi} \sigma D} \exp \left[\frac{-\ln ^{2}\left(D / D_{0}\right)}{2 \sigma^{2}}\right]
$$

Chantrell et al. have developed a numerical calculation method to determine the standard deviation $(\sigma)$ and median particle diameter $(D)$ from the magnetization curve at room temperature [37]. Following that, the magnetization of a noninteracting superparamagnetic nanoparticle system with log-normal distribution of particle sizes in a certain applied magnetic field with weak magnetic anisotropy $\left(K V<<\mathrm{k}_{\mathrm{B}} T\right)$ is given by

$$
\begin{aligned}
M(H) & =M_{s} \int_{0}^{\infty} L \cdot f(y) \cdot \mathrm{d} y \\
& =M_{s} \int_{0}^{\infty} L \cdot\left[\frac{\mathrm{M}_{\mathrm{sb}} V_{m V} H}{k_{B} T} y^{3}\right] F_{V} \cdot \mathrm{d} y,
\end{aligned}
$$

where $M(H)$ and $M_{s}$ are the magnetization and saturation magnetization of superparamagnetic nanoparticle systems, respectively. $L(x)$ is the Langevin function and $f(y)=f(D)$ is the particle size distribution function of the system. $V_{m V}=$ $\pi D_{m V}^{3} / 6$ is the median volume of particles with $D_{m V}$ representing the median diameter in the volume-weighted distribution. $F_{y}(y)$ is the volume-weighted distributions of the reduced diameters.

\section{Results and Discussion}

3.1. X-Ray Diffraction. The results of the X-ray phase analysis on the formation of samples were confirmed by X-ray diffraction (RD), obtained with the D8 AdvanceBruckner diffraction meter (Germany), using $\mathrm{CuK}_{\propto}$ radiation with $\lambda=0.15406 \mathrm{~nm}$ in the range of $2 \theta$ from $10^{\circ}$ to $70^{\circ}$ at room temperature $(300 \mathrm{~K})$. 


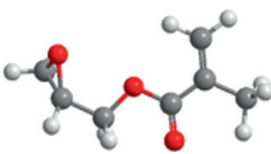

GMA

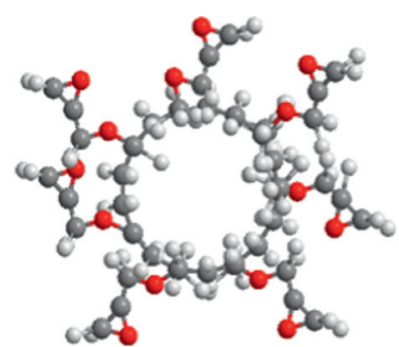

PGMA
Ethylene diamine

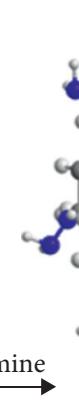

- Nitrogen

- $\mathrm{Fe}_{3} \mathrm{O}_{4}$ nanoparticles
Oxygen

Hydrogen

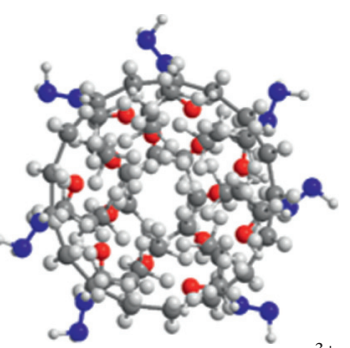

$\mathrm{Fe}^{3+}+\mathrm{Fe}^{2+}+\mathrm{NH}_{3} \cdot \mathrm{H}_{2} \mathrm{O}$

M-PGMA

Figure 1: Overview diagram of the experiment.

Figure 2 shows the XRD pattern of samples: bare $\mathrm{Fe}_{3} \mathrm{O}_{4}$ MNPs (a in Figure 2) and M-PGMA (b in Figure 2). According to X-ray diffraction analysis (Figure 2), the dif-

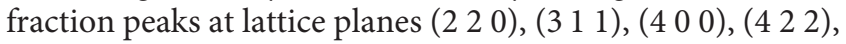
(5 11 1), and (4 400$)$ are well matched with the standard XRD data for magnetite (JCPDS cards no. 75-0033). Moreover, the secondary phases are not found (in Figure $2 b$ ), which means the purified $\mathrm{Fe}_{3} \mathrm{O}_{4}$ MNPs were formed inside the PGMA polymer. However, as mentioned above, it has an approximately poor crystalline structure and peak intensity, caused by the influence of the amorphous PGMA polymer matrix.

The Scherrer equation is used for obtaining the average crystallite sizes $\left(\mathrm{d}_{\mathrm{XRD}}\right)$ of the samples:

$$
d_{X R D}=\frac{K \cdot \lambda}{\beta \cdot \cos \theta},
$$

where $K^{\sim} 0.9$ is the Scherrer constant, $\beta$ is the full width at half maxima of the strongest intensity diffraction peak, $\lambda$ is the wavelength of radiation, and $\theta$ is the angle of the strongest peak. As a result, the crystallite size of the $\mathrm{Fe}_{3} \mathrm{O}_{4}$ MNPs in M-PGMA microspheres is $d_{\mathrm{Fe}_{3} \mathrm{O}_{4}}=3.8$ (nm).

3.2. Fourier Transform Infrared Spectra. The chemical structure of synthesized samples was analyzed by Fourier transform infrared (FTIR) spectra on a Brucker Model IFS 28 spectrometer, which is also one of the most common techniques to identify the attachment of the polymer to the surface of the nanoparticles in nanocomposite materials. Figure 3 shows the FTIR spectra of two samples, PGMA (a in Figure 3 ) and M-PGMA (b in Figure 3), which are measured at room temperature $(300 \mathrm{~K})$ and in the wavenumber range of $400-4000 \mathrm{~cm}^{-1}$.

The FTIR spectra of the pure PGMA (a in Figure 3) show that the characteristic absorption bands observed at $1149 \mathrm{~cm}^{-1}$ and $846 \mathrm{~cm}^{-1}, 993 \mathrm{~cm}^{-1}$ are related to symmetric oxirane ring and asymmetric oxirane ring, respectively. The carbonyl stretching vibrations in the PGMA appear at around $1730 \mathrm{~cm}^{-1}$. In addition, the dip observed at $3930 \mathrm{~cm}^{-1}$ is an alcoholic $(\mathrm{O}-\mathrm{H})$ stretching

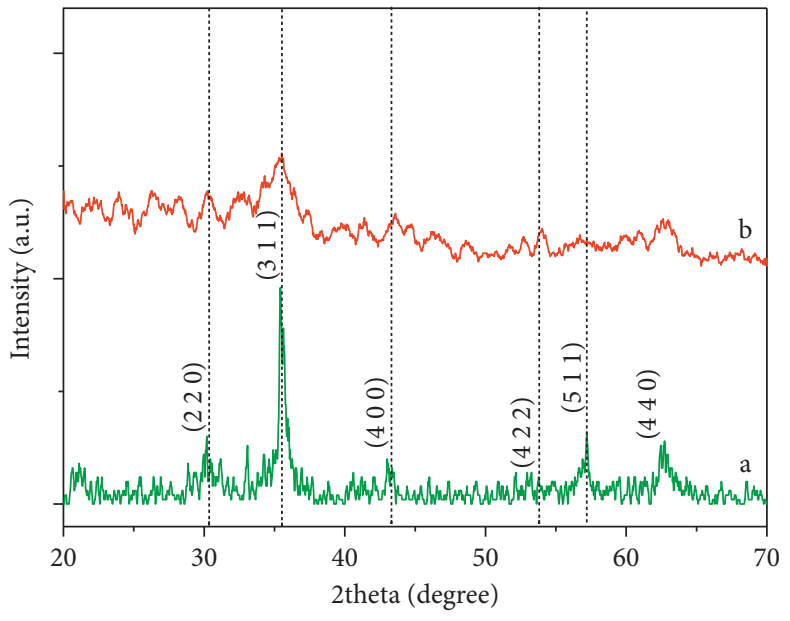

FIgURE 2: X-ray diffraction pattern of (a) bare $\mathrm{Fe}_{3} \mathrm{O}_{4}$ MNPs and (b) M-PGMA.

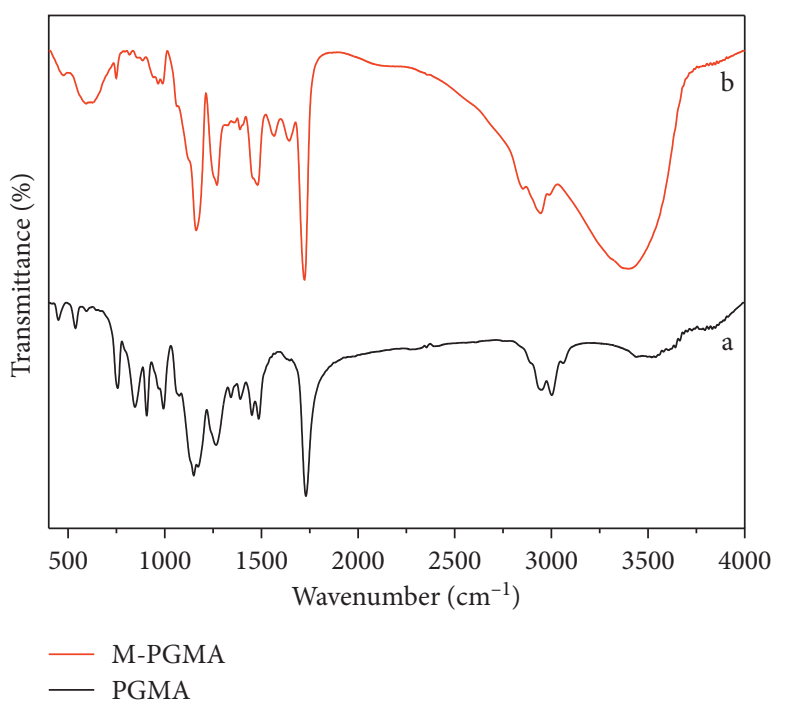

FIgURE 3: The FTIR spectra of two samples in transmittance mode: (a) PGMA and (b) M-PGMA. 
band. However, the FTIR spectra of the M-PGMA (b in Figure 3) showed that the dips located at $846 \mathrm{~cm}^{-1}$ and $993 \mathrm{~cm}^{-1}$ almost disappear, which are characteristic of the asymmetric oxirane ring $\left(\mathrm{O}-\mathrm{CH}_{2}-\mathrm{CH}\right)$. Aside from that, the appearance of new absorption bands at $3399 \mathrm{~cm}^{-1}$, $1566 \mathrm{~cm}^{-1}$, and $1644 \mathrm{~cm}^{-1}$ demonstrates the presence of $\mathrm{NH}$ and $\mathrm{NH}_{2}$ groups, which is the result of modification of the PGMA by ethylenediamine. On the one hand, the presence of a new absorption band observed at $592 \mathrm{~cm}^{-1}$ corresponds to the vibration of the $\mathrm{Fe}-\mathrm{O}$ stretching band, which is related to the existence of the $\mathrm{Fe}_{3} \mathrm{O}_{4}$ MNPs in the PGMA microspheres. On the other hand, the dip observed at $1130 \mathrm{~cm}^{-1}$ corresponding to $(\mathrm{C}-\mathrm{O})$ stretching vibration was shifted to a higher frequency, which has supposed that the PGMA polymer interacted with metal ions of the MNPs and immobilized them; a similar result was discussed in [11].

3.3. Microstructure and Morphology. In order to determine the particle size distribution and morphology of the synthesized material, scanning electron microscopy (SEM, JSM5500 ) at a voltage of $15 \mathrm{kV}$ was carried out. The particle size distribution of the M-PGMA microspheres was determined by measuring the diameter of 100 particles from the SEM images, sorting the sizes into a histogram, and then fitting the histogram data to a log-normal function, which was mentioned in equation (5).

$$
f(D)=\frac{1}{\sqrt{2 \pi} \sigma D} \exp \left[\frac{-\ln ^{2}\left(D / D_{0}\right)}{2 \sigma^{2}}\right],
$$

where $D_{0}$ is the most probable particle diameter and $\sigma$ is the width of the distribution. The median diameter $\langle D\rangle_{\text {SEM }}=D_{0} \cdot \exp \left(\sigma^{2} / 2\right)$ and standard deviation of the distribution $\sigma_{\mathrm{SEM}}=\langle D\rangle_{\mathrm{SEM}} \cdot\left[\exp \left(\sigma^{2}\right)-1\right]^{1 / 2}$.

The SEM images of the M-PGMA microspheres in Figure 4 clearly illustrated the spherical morphology, monodispersed and quite uniform in size. Besides that, their surface is quite rough, which is the result of modification surface of the PGMA by ethylenediamine and because of the presence of the $\mathrm{Fe}_{3} \mathrm{O}_{4}$ MNPs on their surface. The median size of the M-PGMA was $\langle D\rangle_{\mathrm{SEM}}=1.36(\mu \mathrm{m})$ and the corresponding standard deviation $\sigma_{\mathrm{SEM}}=0.09(\mu \mathrm{m})$.

3.4. Magnetic Characterization. Vibrating sample magnetometer (VSM) (MicroSense, USA, Model 029349-A01 $6 \mathrm{MM}$ ) was used to analyze the magnetic properties of the synthesized sample at room temperature $(300 \mathrm{~K})$. A magnetic field of $15 \mathrm{kOe}\left(1.19 \times 10^{6} \mathrm{~A} . \mathrm{m}^{-1}\right)$ was applied.

Figure 5 displays the magnetization curve of the M-PGMA at room temperature, which shows the superparamagnetic behavior. The values of the saturation magnetization $\left(M_{s}\right)$, the remanent magnetization $\left(M_{r}\right)$, and the coercive field $\left(H_{c}\right)$ obtained from the magnetization curve are given in Table 1. When comparing with the bare magnetite nanoparticles and bulk magnetite material, which have the saturation magnetization
$M_{\mathrm{s}} \sim 90-100 \mathrm{emu} / \mathrm{g}$ [38], we noticed a strong decrease in the saturation magnetization of the synthesized material. Li et al. showed that not only the saturation magnetization $\left(M_{s}\right)$ but also the remanent magnetization $\left(M_{r}\right)$ and the coercive field $\left(H_{c}\right)$ of the $\mathrm{Fe}_{3} \mathrm{O}_{4}$ MNPs system are highly influenced by the particle size and domain structure $[17,39]$. The decrease of the magnetism parameters corresponds to decreasing particle size, regardless of the crystal structure and particle shape. According to the result obtained from the XRD pattern shown above, the crystallite size of the $\mathrm{Fe}_{3} \mathrm{O}_{4}$ MNPs in M-PGMA microspheres is $d_{\mathrm{XRD}}=3.8(\mathrm{~nm})$, which had led to reducing the amplitude of the magnetism parameters of the M-PGMA microspheres material. Besides that, the MNPs were immobilized in the PGMA polymer matrix, which formed the noninteracting magnetic nanoparticles system. This leads to a decrease in the dipole-dipole interaction between the MNPs, which contributes to a decrease in the saturation magnetization of the MNPs [40].

To measure zero-field-cooled (ZFC) magnetization curves, first of all, the sample is cooled from $300 \mathrm{~K}$ to $2 \mathrm{~K}$ (without the external applied magnetic field). Thereafter, a weak magnetic field $(50 \mathrm{Oe})$ is applied, and the magnetic moment is recorded with an increase of temperature to obtain the ZFC curve. Under the same 50 Oe applied field but with a bit discrepancy, the field-cooled (FC) curve is measured during the sample cooling from room temperature $(300 \mathrm{~K})$ and magnetic moment is recorded with decreasing temperature.

Figure 6 displays the ZFC/FC magnetization curves of the sample measured at $50(\mathrm{Oe})$. The blocking temperature $\left(T_{\mathrm{B}}\right)$ was obtained directly from the ZFC $M(T)$ magnetization curves, which is the top of the ZFC curve $\left(T_{\mathrm{B}}=10 \mathrm{~K}\right)$. Explaining for $T_{\mathrm{B}}$ obtained at very low temperature, primary cause based on the blocking/unblocking mechanism of the MNPs is having single domain according to their sizes, smaller particles are easily unblocked at lower temperatures. We obtained $K(T)$ from relation with $T_{\mathrm{B}}$ as follows [41]:

$$
K_{\text {eff }}=\frac{25 k_{B} T_{B}}{V},
$$

where $K_{\text {eff }}(T)$ is the effective magnetic anisotropy constant at the blocking temperature $\left(T_{\mathrm{B}}\right), k_{\mathrm{B}}$ is the Boltzmann constant, and $V$ is the median volume of the particles. From that, we can calculate $K_{\text {eff }}=1.9 \times 10^{4}\left(\mathrm{~J} / \mathrm{m}^{3}\right)$, which is close to the value reported by other studies for the bare $\mathrm{Fe}_{3} \mathrm{O}_{4} \mathrm{MNPs}$ (particle size $\sim 5-8 \mathrm{~nm}$ ) and larger than the bulk value for $\mathrm{Fe}_{3} \mathrm{O}_{4}\left(1.1 \times 10^{4} \mathrm{~J} / \mathrm{m}^{3}\right)[17,42-49]$. The primary cause is due to the dependence of the $K_{\text {eff }}$ on the particle size of MNPs ( $K_{\text {eff }}$ increases as the size of MNPs decreases) which has been reported in [50,51]. It is explained by the increase in surface anisotropy as the particle size decreases.

As mentioned above, the LAS principle is used to estimate the effective anisotropy constant $\left(K_{\text {eff }}\right)$ of the synthesized material. We use the reduced magnetization $m=M /$ $M_{s}$, which as a function of $1 / \mathrm{H}^{2}$ was fitted linearly by equation (2) at high magnetic field strengths 


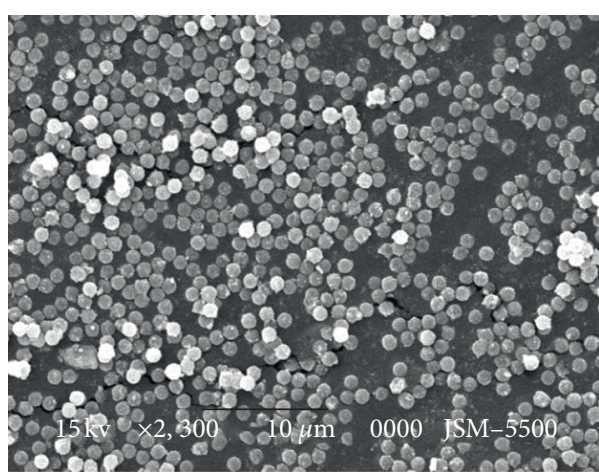

(a)

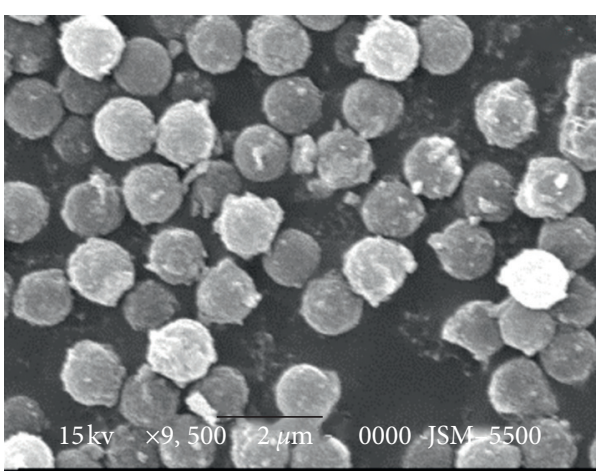

(b)

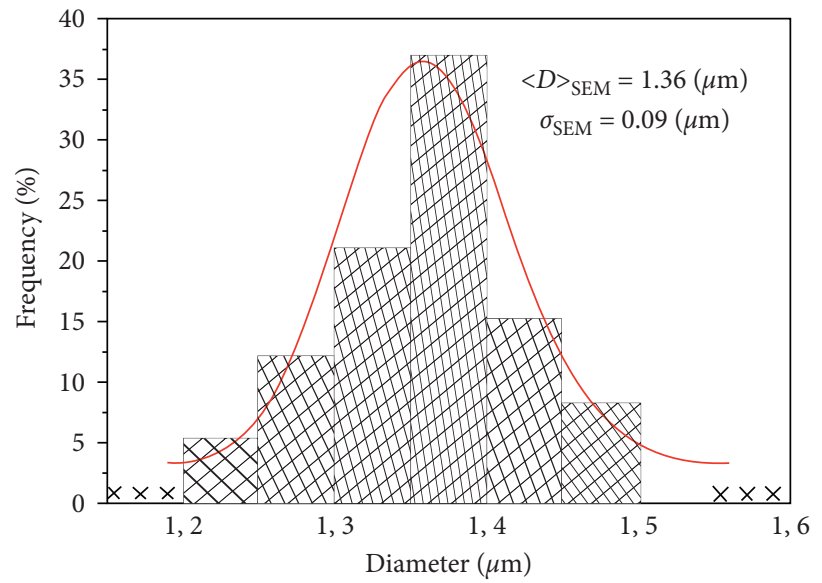

(c)

Figure 4: SEM image of M-PGMA at the two scales: (a) $10 \mu \mathrm{m}$ and (b) $2 \mu \mathrm{m}$. (c) The histogram of particle size distribution fitted with a lognormal function (bold solid lines).

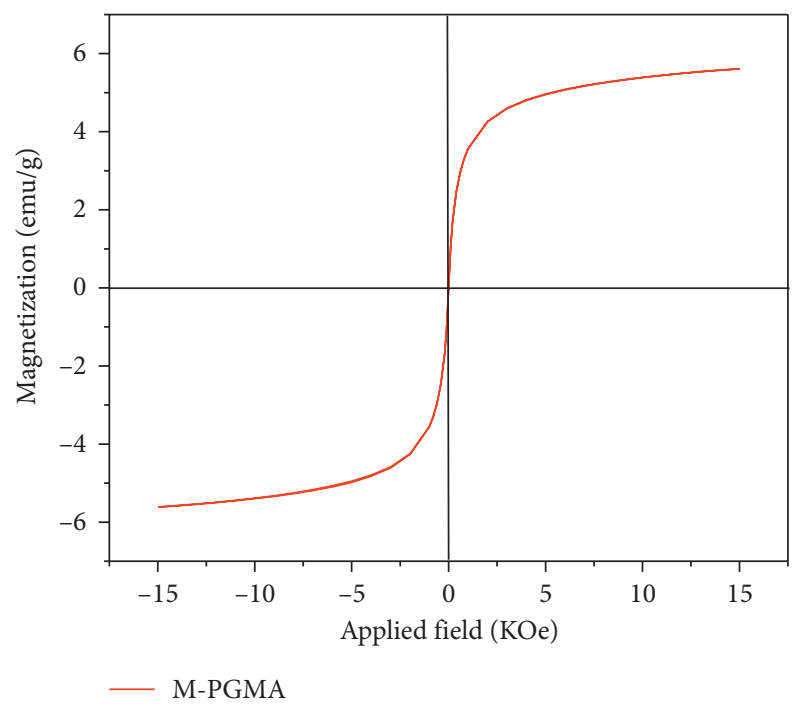

FIgURE 5: The magnetization curves of M-PGMA at room temperature.

$\left(7.96 \times 10^{5}-1.19 \times 10^{6} \mathrm{~A} . \mathrm{m}^{-1}\right)$ (Figure 7$)$. The obtained value $b$ from the slope of the linear fitting is used to calculate $K_{\text {eff }}$ following equation (3). The estimated value of $K_{\text {eff }}$ of the M-PGMA microsphere at room temperature $(300 \mathrm{~K})$ is $K_{\text {eff }}$
$(\mathrm{LAS})=2.9 \pm 0.6 \times 10^{3}\left(\mathrm{~J} / \mathrm{m}^{3}\right)$. Compared to $K_{\text {eff }}\left(T_{\mathrm{B}}\right)$ obtained above, it has a discrepancy in magnitude (displayed in Table 1), which can be understood based on the temperature dependence of magnetic anisotropy constant [52, 53]. As a result, the magnitude of $K_{\text {eff }}$ decreases with increasing temperature, and $K_{\text {eff }}\left(T_{\mathrm{B}}\right)$ obtained at $10 \mathrm{~K}$ is larger than $K_{\text {eff }}$ (LAS) obtained at $300 \mathrm{~K}$.

3.5. Particle Size Distribution Function. When formed inside the M-PGMA microspheres, the MNPs are embedded and immobilized by the PGMA polymer matrix. Therefore, the MNPs system becomes a noninteracting magnetic nanoparticle system.

According to Chantrell et al., the magnetization of a noninteracting superparamagnetic system with a certain distribution of particle sizes (assuming all particles have sphere morphology and the form of the size distribution is log-normal distribution (equation (5)) at room temperature can be used to extract the particle size distribution. The experimentally measured magnetization curve data is used as mentioned above, which is fitted with the Langevin function for both low field data (initial susceptibility) and high field data (approach to saturation) to determine particle size distribution (Figure 8). 
TABle 1: The magnetic parameters of M-PGMA.

\begin{tabular}{lcccc}
\hline$M_{\mathrm{s}}(\mathrm{emu} / \mathrm{g})$ & $H_{\mathrm{c}}(\mathrm{Oe})$ & $\mathrm{M}_{\mathrm{r}}(\mathrm{emu} / \mathrm{g})$ & $\mathrm{K}_{\mathrm{eff}}(\mathrm{LAS})\left(\times 10^{3} \mathrm{~J} \mathrm{~m}^{-3}\right)$ & $\mathrm{K}_{\mathrm{eff}}\left(\mathrm{T}_{\mathrm{B}}\right)\left(\times 10^{4} \mathrm{~J} \mathrm{~m} \mathrm{~m}^{-3}\right)$ \\
\hline 5.61 & 1.42 & 0.015 & $2.9 \pm 0.6$ & $1.9 \pm 0.15$ \\
\hline
\end{tabular}

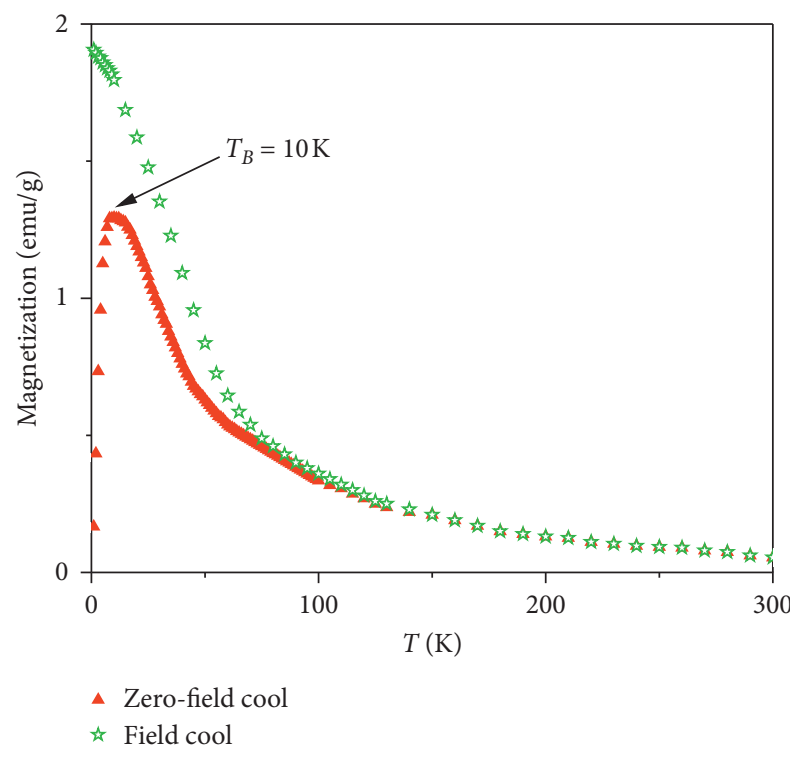

FIGURE 6: ZFC/FC magnetization curves for the M-PGMA microsphere. The cooling field was $50(\mathrm{Oe})$.

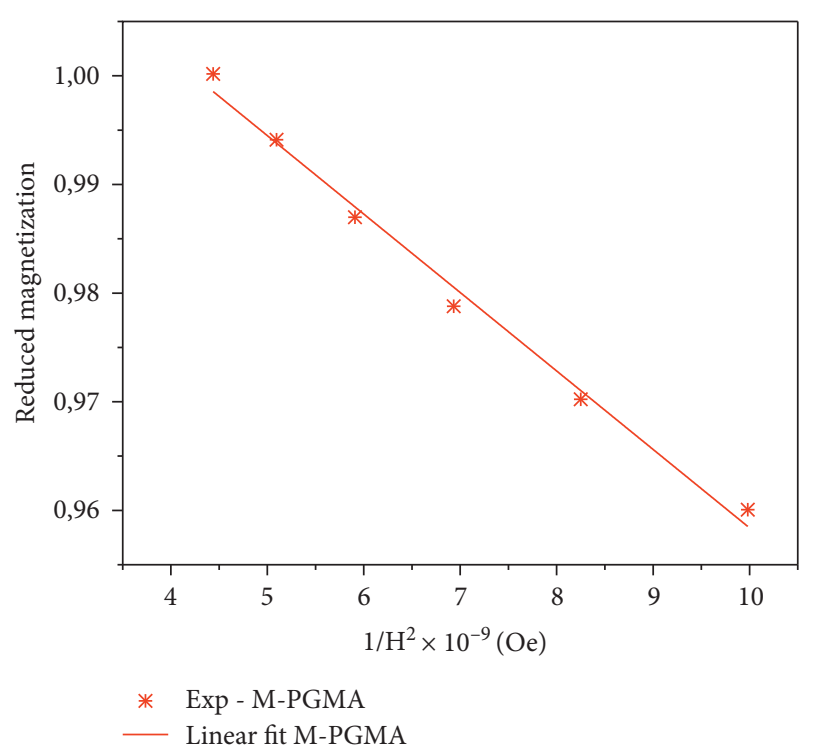

Figure 7: The $M / M_{s}-1 / H^{2}$ dependence of M-PGMA at high field strengths. The solid line represents a linear fit of the experimental data.

From the method of Chantrell et al. [37], we obtained the mean particle size $(D)$ and the standard deviation $\left(\sigma_{\mathrm{D}}\right)$, respectively:

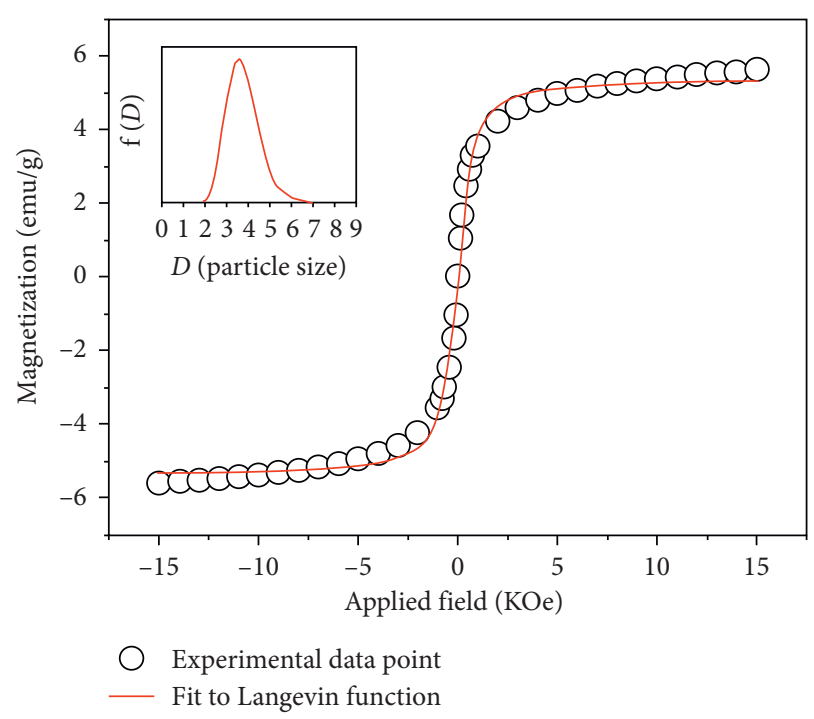

Figure 8: The magnetization curve of M-PGMA at room temperature. The solid line shows the fit to the $M(H)$ curve using Langevin function. The top inset shows the particle size distribution function.

$$
\begin{aligned}
D & =\left[\frac{18 k_{B} T}{\pi \mathrm{M}_{\mathrm{sb}}} \sqrt{\frac{\chi_{i}}{3 \mathrm{M}_{\mathrm{s}} \cdot H_{0}}}\right]^{\frac{1}{3}}, \\
\sigma_{D} & =\frac{1}{3} \sqrt{\ln \left(\frac{3 \chi_{i} H_{0}}{\mathrm{M}_{\mathrm{s}}}\right)},
\end{aligned}
$$

where $D$ is the mean particle size of the MNPs inside magnetic poly (glycidyl methacrylate) microspheres, $\chi_{i}$ is the initial susceptibility, $M_{\mathrm{s}}$ is the saturation magnetization of the particle, $M_{\mathrm{sb}}$ is the saturation magnetization of the bulk material, $T$ is the room temperature in Kelvin $(300 \mathrm{~K}), H_{0}$ is the certain applied field at high field corresponding to the magnetization reduced to zero, and $k_{\mathrm{B}}$ is the Boltzmann constant $\left(1.38 \times 10^{-23} \mathrm{~J} \mathrm{~K}^{-1}\right)$.

From that, we obtain $D=3.76(\mathrm{~nm}), \sigma_{\mathrm{D}}=0.77(\mathrm{~nm})$, and the particle size distribution function in Figure 8 (topset). This shows a narrow distribution in size, and the MNPs formed in the M-PGMA microspheres have a high uniformity in size, which is in excellent agreement with the average crystallite size determined by XRD ( $\left.D_{\mathrm{XRD}} \sim 3.8 \mathrm{~nm}\right)$. It is demonstrated that the MNPs embedded inside the PGMA microsphere have a monocrystalline structure, with single domain. Besides that, the particle size distribution function has good agreement with the anisotropy energy barrier distribution displayed in the ZFC magnetization curve (lower part of the ZFC curve). 


\section{Conclusions}

In this work, we have investigated the characteristics of the M-PGMA microspheres synthesized material by various experimental analysis methods. The synthesized magnetic microsphere material are monodispersed and quite uniform in size. Their median size and corresponding standard deviation were $\langle D\rangle_{\mathrm{SEM}}=1.36(\mu \mathrm{m})$ and $\sigma_{\mathrm{SEM}}=0.09(\mu \mathrm{m})$, respectively. The purified $\mathrm{Fe}_{3} \mathrm{O}_{4} \mathrm{MNPs}$ were formed inside the PGMA polymer matrix, which has a monocrystalline structure, single domain, median particle size $D=3.76(\mathrm{~nm})$, corresponding standard deviation $\sigma_{\mathrm{D}}=0.77(\mathrm{~nm})$, and particle size distribution function with a narrow distribution in size. Magnetic properties of the M-PGMA microspheres have also been investigated, which have superparamagnetic behavior and the magnetism parameters which are the saturation magnetization $\left(M_{\mathrm{s}}\right)$, the remanent magnetization $\left(M_{\mathrm{r}}\right)$, and the coercive field $\left(H_{\mathrm{c}}\right)$ are $5.16(\mathrm{emu} / \mathrm{g}), 0.015$ (emu/g), and $1.42(\mathrm{Oe})$, respectively. Besides that, the $K_{\text {eff }}$ of the synthesized material was determined by two different methods: ZFC/FC measurement at $10 \mathrm{~K}$ (experimental method) and LAS principle at $300 \mathrm{~K}$ (theoretically approach) with the obtained values $K_{\text {eff }}\left(T_{\mathrm{B}}\right)=1.9 \pm 0.15 \times 10^{4}\left({\left.\mathrm{~J} . \mathrm{m}^{-3}\right)}^{-3}\right.$ and $K_{\text {eff }}(\mathrm{LAS})=2.9 \pm 0.6 \times 10^{3}\left(\mathrm{~J} . \mathrm{m}^{-3}\right)$, respectively. By investigating $K_{\text {eff }}$, the influence of the PGMA polymer matrix on the MNPs embedded inside was considered. The PGMA polymer molecule interacted with surface atoms of the MNPs immobilized inside the M-PGMA microspheres forming a magnetically disordered shell or nonmagnetic layer. On the one hand, this decreased the magnetic core size of the MNPs. On the other hand, it had led to an increase in the surface anisotropy and a reduction of interparticle interactions (dipole-dipole interaction). As a result, it not only reduces the magnetic parameters including $M_{\mathrm{s}}, M_{\mathrm{r}}$, and $H_{\mathrm{c}}$ but also increases the magnetic anisotropy of the synthesized nanocomposite material.

\section{Data Availability}

The data used to support the findings of this study are included within the article.

\section{Conflicts of Interest}

The authors declare that they have no conflicts of interest.

\section{References}

[1] C. Anushree and J. Philip, "Efficient removal of methylene blue dye using cellulose capped $\mathrm{Fe}_{3} \mathrm{O}_{4}$ nanofluids prepared using oxidation-precipitation method," Colloids and Surfaces A: Physicochemical and Engineering Aspects, vol. 567, pp. 193-204, 2019.

[2] C. Anushree, D. N. G. Krishna, and J. Philip, "Oil-absorbent MnOx capped iron oxide nanoparticles: synthesis, characterization and applications in oil recovery," Journal of $\mathrm{Mo}$ lecular Liquids, vol. 320, Article ID 114324, 2020.

[3] S. Arsalani, E. J. Guidelli, M. A. Silveira et al., "Magnetic $\mathrm{Fe}_{3} \mathrm{O}_{4}$ nanoparticles coated by natural rubber latex as MRI contrast agent," Journal of Magnetism and Magnetic Materials, vol. 475, pp. 458-464, 2019.
[4] A. Sohail, Z. Ahmad, O. A. Bég, S. Arshad, and L. Sherin, "A review on hyperthermia via nanoparticle-mediated therapy," Bulletin du Cancer, vol. 104, no. 5, pp. 452-461, 2017.

[5] P. Y. Yen, M. M. KamyarShameli et al., "Green biosynthesis of superparamagnetic magnetite $\mathrm{Fe}_{3} \mathrm{O}_{4}$ nanoparticles and biomedical applications in targeted anticancer drug delivery system: a review," Arabian Journal of Chemistry, vol. 13, pp. 2287-2308, 2020.

[6] M. Shao, F. Ning, J. Zhao, M. Wei, D. G. Evans, and X. Duan, "Preparation of $\mathrm{Fe}_{3} \mathrm{O}_{4} @ \mathrm{SiO}_{2} @$ Layered double hydroxide core-shell microspheres for magnetic separation of proteins," Journal of the American Chemical Society, vol. 134, no. 2, pp. 1071-1077, 2012.

[7] S. K. Prajapati, A. Jain, A. Jain, and S. Jain, "Biodegradable polymers and constructs: a novel approach in drug delivery," European Polymer Journal, vol. 120, Article ID 109191, 2019.

[8] I. M. Lourenço, M. T. Pelegrino, J. C. Pierettil, G. P. Andrade, G. Cerchiaro, and A. B. Seabra, "Synthesis, characterization and cytotoxicity of chitosan-coated $\mathrm{Fe}_{3} \mathrm{O}_{4}$ nanoparticles functionalized with ascorbic acid for biomedical applications," Journal of Physics: Conference Series, vol. 1323, 2015.

[9] E. Calzoni, A. Cesaretti, A. Polchi, A. D. Michele, B. Tancini, and C. Emiliani, "Biocompatible polymer nanoparticles for drug delivery applications in cancer and neurodegenerative disorder therapies," Journal of Functional Biomaterials, vol. 10, no. 1, p. 4, 2019.

[10] H. Kaplan Can, S. Kavlak, S. ParviziKhosroshahi, and G. Ali, "Preparation, characterization and dynamical mechanical properties of dextran-coated iron oxide nanoparticles (DIONPs)," Artificial Cells, Nanomedicine, and Biotechnology, vol. 46, no. 2, 2018.

[11] J. Koubková, P. Müller, H. Hlídková et al., "Magnetic poly (glycidyl methacrylate) microspheres for protein capture," New Biotechnology, vol. 31, no. 5, pp. 482-491, 2014.

[12] Q. L. Li, W. X. Gu, H. Gao, and Y. W. Yang, "Self-assembly and applications of poly (glycidyl methacrylate) s and their derivatives," Chemical Communications, vol. 50, p. 1320, 2014.

[13] Z. B. Zhao, L. Tai, D.-M. Zhang, and Y. Jiang, "Facile fabrication of siloxane @ poly (methylacrylic acid) core-shell microparticles with different functional groups," Journal of Nanoparticle Research, vol. 19, no. 2, p. 73, 2017.

[14] L. Cheng, J. Wu, H. Liang, and Q. Yuan, "Preparation of poly (glycidyl methacrylate) (PGMA) and amine modified PGMA adsorbents for purification of glucosinolates from cruciferous plants," Molecules, MDPI, vol. 25, no. 14, p. 3286, 2020.

[15] Y. Yuan, D. Rende, C. L. Altan, S. Bucak, R. Ozisik, and T. D. A. Borca, "Effect of surface modification on magnetization of iron oxide nanoparticle colloids," Langmuir, American Chemical Society, vol. 28, no. 36, pp. 13051-13059, 2012.

[16] K. Dukenbayev, I. Korolkov, D. Tishkevich et al., " $\mathrm{Fe}_{3} \mathrm{O}_{4}$ nanoparticles for complex targeted delivery and boron neutron capture therapy," Nanomaterials, vol. 9, no. 4, pp. 494-512, 2019.

[17] G. Gnanaprakash, J. Philip, T. Jayakumar, and B. Raj, "Effect of digestion time and alkali addition rate on physical properties of magnetite nanoparticles," The Journal of Physical Chemistry B, vol. 111, no. 28, pp. 7978-7986, 2007.

[18] P. Tartaj, M. del Puerto Morales, S. Veintemillas-Verdaguer, T. González-Carreño, and C. J. Serna, "The preparation of magnetic nanoparticles for applications in biomedicine," Journal of Physics D Applied Physics, vol. 36, no. 13, p. 182, 2003. 
[19] S. Laurent, D. Forge, M. Port et al., "Magnetic iron oxide nanoparticles: synthesis, stabilization, vectorization, physicochemical characterizations, and biological applications," Chemistry Review, vol. 108, no. 6, pp. 2064-2110, 2008.

[20] C. Anushree, D. N. G. Krishna, B. B. Lahiri, and J. Philip, "Enhanced thermal protection of iron oxide nanoparticle by insulating nanoporous char layer: effect of core size and char layer properties," Journal of Physical Chemistry C, vol. 124, no. 10 , pp. $5702-5714,2020$.

[21] D. Horák and N. Chekina, "Preparation of magnetic poly (glycidyl methacrylate) microspheres by emulsion polymerization in the presence of sterically stabilized iron oxide nanoparticles," Journal of Applied Polymer science, vol. 102, no. 5, pp. 4348-4357, 2006.

[22] A. Z. Beata, H. Helena, P. Eduard et al., "Monodisperse magnetic poly (glycidyl methacrylate) microspheres for isolation of autoantibodies with affinity for the $46 \mathrm{kDa}$ form of unconventional Myo1C present in autoimmune patients," Microchimica Acta, vol. 185, no. 5, 2018.

[23] B. Medasani and I. Vasiliev, "Computational study of the surface properties of aluminum nanoparticles," Surface Science, vol. 603, no. 13, pp. 2042-2046, 2009.

[24] L. I. Conde, D. Baldomir, B. C. Martinez et al., "A single picture explains diversity of hyperthermia response of magnetic Nanoparticles," Journal of Physical Chemistry C, vol. 119, no. 27, pp. 15698-15706, 2015.

[25] J. P. Fortin, C. Wilhelm, J. Servais, C. Ménager, J. C. Bacri, and F. Gazeau, "Size-sorted anionic iron oxide nanomagnets as colloidal mediators for magnetic hyperthermia," Journal of the American Chemical Society, vol. 129, no. 9, pp. 2628-2635, 2007.

[26] Z. Nemati, J. Alonso, I. Rodrigo et al., "Improving the heating efficiency of iron oxide nanoparticles by tuning their shape and size," Journal of Physical Chemistry C, vol. 122, no. 4, pp. 2367-2381, 2018.

[27] Z. Nemati, J. Alonso, L. M. Martinez et al., "Enhanced magnetic hyperthermia in iron oxide nano-octopods: size and anisotropy effects," Journal of Physical Chemistry C, vol. 120, no. 15, pp. 8370-8379, 2016.

[28] G. C. Lavorato, R. Das, Y. Xing et al., "Origin and shell-driven optimization of the heating power in core/shell bimagnetic nanoparticles," ACS Applied Nano Materials, vol. 3, no. 2, pp. 1755-1765, 2020.

[29] R. C. Woodward, J. Heeris, T. G. S. Pierre et al., "A Comparison of methods for the measurement of the particle-size distribution of magnetic nanoparticles," Journal of Applied Crystallography, vol. 40, pp. 495-500, 2007.

[30] S. Chikazumi, Physics of Ferromagnetism, Oxford University Press, Oxford, UK, 1997.

[31] W. F. Brown, "Theory of the approach to magnetic saturation," Physics Review, vol. 58, pp. 736-743, 1940.

[32] H. Hauser, D. C. Jiles, Y. Melikhov, L. Li, and R. Grössinger, "An approach to modeling the dependence of magnetization on magnetic field in the high field regime," Journal of Magnetism and Magnetic Materials, vol. 300, no. 2, pp. 273283, 2006.

[33] Z. Nemati, S. M. Salili, J. Alonso et al., "Superparamagnetic iron oxide nanodiscs for hyperthermia therapy: does size matter?" Journal of Alloys and Compounds, vol. 714, pp. 709-714, 2017.

[34] M. Knobel, W. C. Nunes, L. M. Socolovsky et al., "Superparamagnetism, other magnetic, features in granular materials: a review on ideal and real systems," Journal of Nanoscience and Nanotechnology, vol. 8, pp. 2836-2857, 2008.
[35] J. Heintzenberg, "Properties of the log-normal particle size distribution," Aerosol Science and Technology, vol. 21, no. 1, pp. 46-48, 1994.

[36] D. Henrard, Q. L. Vuong, S. Delangre et al., "Monitoring of superparamagnetic particle sizes in the Langevin law regime," Journal of Nanomaterials, vol. 2019, Article ID 6409210, 9 pages, 2019.

[37] R. Chantrell, J. Popplewell, and S. Charles, "Measurements of particle size distribution parameters in ferrofluids," IEEE Transactions on Magnetics, vol. 14, no. 5, pp. 975-977, 1978.

[38] K. Nishio, M. Ikeda, S. Tsubouchi et al., "Preparation of sizecontrolled (30-100 nm) magnetite nanoparticles for biomedical applications," Journal of Magnetism and Magnetic Materials, vol. 310, no. 2, pp. 2408-2410, 2007.

[39] Q. Li, W. C. Kartikowati, S. Horie, T. Ogi, T. Iwaki, and K. Okuyama, "Correlation between particle size/domain structure and magnetic properties of highly crystalline $\mathrm{Fe}_{3} \mathrm{O}_{4}$ nanoparticles," Scientific Reports, vol. 7, 2017.

[40] D. Peddis, O. Federica, A. Ardu, C. Cannas, A. Musinu, and G. Piccaluga, "Interparticle interactions and magnetic anisotropy in cobalt ferrite nanoparticles: influence of molecular coating," Chemistry of Materials, vol. 24, no. 6, pp. 1062-1071, 2012.

[41] P. Guardiaa, B. B. Brugala, A. G. Rocab et al., "Surfactant effects in magnetite nanoparticles of controlled size," Journal of Magnetism and Magnetic Materials, vol. 316, no. 2, pp. e756-e759, 2007.

[42] D. Klaus, Handbook of Nanophysics: Nanomedicine and Nanorobotics, Vol. 23, Taylor and Francis, Oxford, UK, 2010.

[43] H. Mamiya, H. Fukumoto, H. J. L. Cuya, K. Suzuki, H. Miyamura, and J. Balachandran, "Estimation of magnetic anisotropy of individual magnetite nanoparticles for magnetic hyperthermia," ACS Nano, vol. 14, no. 7, pp. 8421-8432, 2020.

[44] C. Nayek, K. Manna, A. A. Imam, A. Y. Alqasrawi, and I. M. Obaidat, "Size-dependent magnetic anisotropy of PEG coated $\mathrm{Fe}_{3} \mathrm{O}_{4}$ nanoparticles; comparing two magnetization methods," in Proceedings of the 2nd International Conference on International Materials IOP Conference Series: Materials Science and Engineering, vol. 305, Article ID 012012, Irbid, Jordan, July-2017.

[45] R. E. Rosensweig, "Heating magnetic fluid with alternating magnetic field," Journal of Magnetism and Magnetic Materials, vol. 252, pp. 370-374, 2002.

[46] G. F. Goya, T. S. Berquó, F. C. Fonseca, and M. P. Morales, "Static and dynamic magnetic properties of spherical magnetite nanoparticles," Journal of Applied Physics, vol. 94, no. 5, pp. 3520-3528, 2003.

[47] R. M. Ferguson, K. R. Minard, A. P. Khandhar, and K. M. Krishnan, "Optimizing magnetite nanoparticles for mass sensitivity in magnetic particle imaging," Medical Physics, vol. 38, pp. 1619-1626, 2003.

[48] R. Frison, G. Cernuto, A. Cervellino et al., "Magnetite-Maghemite nanoparticles in the $5-15 \mathrm{~nm}$ range: correlating the core-shell composition and the surface structure to the magnetic properties. A total scattering study," Chemistry of Materials, vol. 25, no. 23, pp. 4820-48272, 2013.

[49] B. Mehdaoui, A. Meffre, L.-M. Lacroix, J. Carrey et al., "Magnetic anisotropy determination and magnetic hyperthermia properties of small Fe nanoparticles in the superparamagnetic regime," Journal of Applied Physics, vol. 107, no. 9, p. 324, 2010.

[50] A. F. Lehlooh, S. H. Mahmood, and J. M. Williams, "On the particle size dependence of the magnetic anisotropy energy 
constant," Physica B: Condensed Matter, vol. 321, no. 1-4, pp. 159-162, 2002.

[51] C. Nayek, K. Manna, A. A. Imam, A. Y. Alqasrawi, and I. M. Obaidat, "Size-dependent magnetic anisotropy of PEG coated $\mathrm{Fe}_{3} \mathrm{O}_{4}$ nanoparticles; comparing two magnetization methods," in Proceedings of the 2nd International Conference on International Materials IOP Conference Series: Materials Science and Engineering, vol. 305, Article ID 012012, Irbid, Jordan, July 2017.

[52] A. Franco and V. Zapf, "Temperature dependence of magnetic anisotropy in nanoparticles of $\mathrm{Co}_{\mathrm{x}} \mathrm{Fe}_{(3-\mathrm{x})} \mathrm{O}_{4}$, "Journal of Magnetism and Magnetic Materials, vol. 320, no. 5, pp. 709713,2008 .

[53] J. Wang, H. Duan, X. Lin, V. Aguilar, A. Mosqueda, and G. M. Zhao, "Temperature dependence of magnetic anisotropy constant in iron chalcogenide $\mathrm{Fe}_{3} \mathrm{Se}_{4}$ : excellent agreement with theories," Journal of Applied Physics, vol. 112, no. 10, Article ID 103905, 2012. 